\title{
ASPECTOS FARMACOECONÔMICOS DO TRATAMENTO HORMONAL PARA CÂNCER DE MAMA EM UMA UNIDADE DE ALTA COMPLEXIDADE EM ONCOLOGIA DE RECIFE
}

\author{
A.A.A. Rego $^{1}$, M.F. Cordeiro ${ }^{2}$, M.J.B.M, Rego ${ }^{3}$. \\ ${ }^{1}$ Universidade Federal de Pernambuco, Hospital das Clínicas \\ ${ }^{2}$ Universidade Federal de Pernambuco, Laboratório de Imunomodulação e Novas \\ Abordagens Terapêuticas \\ ${ }^{3}$ Universidade Federal de Pernambuco, CCB, Departamento de Bioquímica \\ E-mail para contato: ariane_alencar@hotmail.com
}

\begin{abstract}
RESUMO - O câncer é um problema de saúde pública mundial. O custo com o tratamento do câncer de mama inclui a hormonioterapia como um dos tratamentos sistêmicos de longa duração e, está entre as medidas terapêuticas com maior controle da doença. Portanto, o objetivo desse trabalho foi realizar uma avaliação farmacoeconômica do tratamento hormonal para o câncer de mama no Hospital das Clínicas de Pernambuco. $O$ número de prescrições dispensadas por tipo de hormônio a nível de farmácia ambulatorial HC/UFPE no periodo de agosto de 2016 a julho de 2017 foi de 1.562 e o custo direto da dispensação de $R \$ 78.432,00$. A quantidade de procedimentos cobrados em Autorização de Procedimentos de Alta Complexidade (APAC) pelo HC/UFPE, referente à hormonioterapia do câncer de mama foi de 1.498 e o valor reembolsado foi de $R \$ 220.805,25$. Conclui-se que o hospital mantém o processo regular de emissão de $A P A C$, o que garante o retorno financeiro referente à terapia hormonal do câncer de mama.
\end{abstract}

Palavras-chave: Câncer de Mama, Hormonioterapia, Custo.

ABSTRACT - Cancer is a global public health problem. The cost of breast cancer treatment includes hormone therapy as one of the long-term systemic treatments and it is, among the therapeutic measures with greater control of the disease. Therefore, the aim of this study was to perform a pharmacoeconomic evaluation of hormonal treatment for breast cancer at the Hospital das Clinicas de Pernambuco. The number of prescriptions dispensed by type of hormone at HC / UFPE outpatient pharmacy in the period from August 2016 to July 2017 was 1,562 and the direct cost of dispensing was $R \$ 78,432.00$. The number of procedures charged in High Complexity Procedures Authorization (HPCA) by the HC / UFPE for the hormone therapy of breast cancer was 1,498 and the amount reimbursed was $R \$ 220,805.25$. It is concluded that the hospital maintains the regular process of issuing HPCA, which guarantees the financial return related to the hormonal therapy of breast cancer.

Keywords: Breast cancer, hormone therapy, cost.

\section{INTRODUÇÃ̃O}


O câncer é um problema de saúde pública mundial, configurando uma das principais causas de mortes no mundo. Por outro lado, a terapia para o tratamento do câncer também tem avançado e melhorado a qualidade de vida dos pacientes, mesmo nos casos decorrentes de metástases (OMS, 2014).

O custo com o tratamento do câncer de mama inclui a hormonioterapia, um dos tratamentos sistêmicos para o câncer de mama, determinado de acordo com o risco de recorrência, e baseia-se na utilização de antagonistas dos hormônios que estimulam o crescimento do tumor. Por isto, a avaliação tumoral deve apresentar receptores para os hormônios estrogênio e progesterona, e tem como objetivo redução de recidiva e melhoria na sobrevida das pacientes (BRASIL, 2008).

Levando-se em conta o fator contributivo da hormonioterapia no aumento da sobrevida das mulheres com câncer de mama e o gasto expressivo de recursos públicos envolvidos por ser um tratamento de longa duração (5 a 10 anos), a utilização destes medicamentos requer planejamento rigoroso no que diz respeito à aquisição e gestão orçamentária. Portanto, o objetivo deste trabalho foi avaliar os aspectos farmacoeconômicos do tratamento hormonal para o câncer de mama em uma unidade de alta complexidade em oncologia de Recife.

\section{MATERIAIS E MÉTODOS}

Foi realizada uma análise nas prescrições dispensadas no período retrospectivo de 12 meses (agosto de 2016 a julho de 2017), pertinentes a todas as mulheres tratadas com hormonioterapia no HC/UFPE durante esse período.

Para análise de custo foi visto o custo direto do tratamento farmacoterapêutico, através do custo unitário dos medicamentos utilizados, adquiridos através de licitação, utilizando como fonte de informação o sistema de controle de estoque utilizado pelo hospital.

Posteriormente, foi feito um comparativo com os valores reembolsados pelo SUS através da cobrança efetuada via Sistema de Informações Ambulatoriais do Sus (SIA/SUS) - APAC/ONCO, através do TabWin, ferramenta de tabulação e tratamento de dados que integra o Datasus, ambos de domínio público.

\section{RESULTADOS E DISCUSSÃO}

Os medicamentos são prescritos de acordo com protocolo vigente, conforme estadiamento da doença, e está em concordância com o Manual de Protocolos Clínicos e Diretrizes Terapêuticas (PCDT) em Oncologia do Ministério da Saúde (MS) (Tabela 1). A terapia é eleita conforme o status menopausal e o risco de recorrência da doença. O Tamoxifeno pode ser utilizado por mulheres em pré ou pós-menopausa. Os inibidores da aromatase (IA), Anastrozol e Exemestano, são utilizados por mulheres em pósmenopausa. 
Tabela 1 - Prescrições dispensadas na Farmácia Ambulatorial e custo da dispensação para o tratamento do câncer de mama no HC|UFPE no período de agosto de 2016 a julho de 2017.

\begin{tabular}{lccc}
\hline Medicamento & $\begin{array}{c}\text { Quantidade } \\
\text { dispensada }\end{array}$ & $\begin{array}{c}\text { Custo unitário da } \\
\mathrm{cx} / 30 \text { comprimidos }\end{array}$ & $\begin{array}{c}\text { Valor da dispensação } \\
\text { (cx/30 comprimidos/paciente) }\end{array}$ \\
\hline $\begin{array}{c}\text { Anastrozol } \\
\text { (cx/30 comprimidos) } \\
\begin{array}{c}\text { Exemestano } 25 \mathrm{mg} \\
\text { (cx/30 comprimidos) } \\
\text { Tamoxifeno 20mg }\end{array}\end{array}$ & 586 & $\mathrm{R} \$ 33,00$ & $\mathrm{R} \$ 19.338,00$ \\
$\begin{array}{l}\text { (cx/30 comprimidos) } \\
\text { Total }\end{array}$ & 908 & $\mathrm{R} \$ 508,50$ & $\mathrm{R} \$ 34.578,00$ \\
\hline
\end{tabular}

Para a cobrança dos procedimentos realizados, são definidos os valores, conforme o estadiamento da doença. Para o tratamento adjuvante hormonal existem três procedimentos: E-I: clínico ou patológico, receptor positivo (R\$ 79,75); E-II: clínico ou patológico com ou sem linfonodos, receptor positivo (R\$ 79,75) e E-III: (ou receptor positivo ou pos-menopausa ( $\mathrm{R} \$ 79,75)$. Para o tratamento paliativo existem dois procedimentos: Mama hormônio $1^{\mathrm{a}}$ linha $(\mathrm{R} \$ 79,75)$; doença óssea ou de partes moles meta ou recidivada; Mama hormônio $2^{\mathrm{a}}$ linha exclusiva pós-menopausa; doença óssea ou de partes moles meta ou recidivada ( $\mathrm{R} \$ 301,50)$, conforme descrito no Manual de Bases Técnicas do Sistema de Informações Ambulatoriais - SIA/SUS (Tabela 2).

Tabela $2-\mathrm{N}^{\mathrm{o}}$ de procedimentos de hormonioterapia cobradas em APAC no HC|UFPE e valores aprovados através do sistema APACnet no período de agosto de 2016 a julho de 2017. Fonte: SIA/SUS em 29/09/17.

\begin{tabular}{l|c|c}
\hline \multicolumn{1}{c|}{ Procedimento APAC/ONCO } & $\begin{array}{c}\text { Quantidade } \\
\text { cobrada em APAC }\end{array}$ & $\begin{array}{c}\text { Valor } \\
\text { aprovado para } \\
\text { reembolso }\end{array}$ \\
\hline $\begin{array}{l}\text { 0304050040 Hormonioterapia do } \\
\text { carcinoma de mama em estádio I }\end{array}$ & 222 & $17.704,50$ \\
$\begin{array}{l}\text { 0304050121 Hormonioterapia do } \\
\text { carcinoma de mama em estádio II }\end{array}$ & 158 & $12.600,50$ \\
$\begin{array}{l}\text { 0304050113 Hormonioterapia do } \\
\text { carcinoma de mama em estádio III } \\
\text { 0304020346 Hormonioterapia do } \\
\text { carcinoma de mama avançado- 1 linha }\end{array}$ & 100 & $7.975,00$ \\
$\begin{array}{l}\text { 0304020338 Hormonioterapia do } \\
\text { carcinoma de mama avançado - 2 }\end{array}$ & 561 & $44.739,75$ \\
$\quad$ Total & 457 & $137.785,50$ \\
\end{tabular}

Do total de prescrições dispensadas versus procedimentos aprovados em APAC, pode-se observar que os custos com a dispensação da hormonioterapia para câncer de mama são cobertos pelos valores de reembolso. Apesar do valor do Exemestano $(\mathrm{R} \$ 508,50)$ ser superior ao maior valor de procedimento repassado $(\mathrm{R} \$ 301,50)$, o custo total é coberto devidoa mudança de linha de tratamento, a mudança do status menopausal, ou quando há recidiva da doença.

Em estudo realizado em hospital beneficente do Paraná, dos 20 procedimentos mais realizados, que representavam 53\% do total de procedimentos efetuados no hospital, teve como resultado que em $90 \%$ dos procedimentos analisados, o valor repassado pelo 
SUS é superior ao custo estimado médio apurado para estes procedimentos (KOS et al, 2015).

Outro estudo realizado em hospital de São Paulo, a nível ambulatorial, concluiu que os valores repassados pelo SUS para o tratamento do câncer colorretal metastático, não cobre nem mesmo os valores dos medicamentos das terapias de $1^{\mathrm{a}}$ e $2^{\mathrm{a}}$ linhas, e portanto, os custos com medicamentos utilizados em oncologia vem gerando grande impacto no orçamento sanitário (TONON, 2007).

\section{CONCLUSÕES}

Os valores reembolsados em APAC referente ao Tamoxifeno e ao Anastrozol cobrem os custos de aquisição desses medicamentos, porém, o maior valor de procedimento, não cobre o custo com aquisição do Exemestano. Entretanto, o total de procedimentos reembolsados cobre o valor total de aquisição dos medicamentos dispensados. Diante do exposto, observa-se que mesmo sendo um tratamento longo, o hospital consegue manter um processo regular de emissão de APAC, o que garante o retorno financeiro referente à terapia hormonal do câncer de mama.

\section{REFERÊNCIAS}

BRASIL, MS. Secretaria de Atenção à Saúde. Protocolos Clínicos e Diretrizes Terapêuticas em Oncologia. Brasília. 2014.

BRASIL, MS. Secretaria de Atenção à Saúde. Instituto Nacional de Câncer. Coordenação de Prevenção e Vigilância de Câncer. Estimativa 2008. Rio de Janeiro: INCA, 2008a. Disponível

em: $<$ http://www.inca.gov.br/estimativa/2008/index.asplink=conteudo_view.asp\&ID=5> Acesso em: 15 de novembro de 2016.

BRASIL, MS. Secretaria de Atenção à Saúde. Departamento de Regulação, Avaliação e Controle Coordenação-geral de Sistemas de Informação. Sistema de Informações Ambulatoriais. Manual de Bases Técnicas em Oncologia. Brasília, 2016.

KOS, S.R.; SANTOS, N.P.; KLEIN, L.; SCARPIN, J.E. Repasse do SUS vs custo dos procedimentos hospitalares: É possível cobrir os custos com o repasse do SUS? XXII Congresso Brasileiro de Custos. Foz do Iguaçu. 2015.

ORGANIZAÇÃO MUNDIAL DE SAÚDE, OMS. Câncer. Disponível em: http://www.who.int/mediacentre/factsheets/fs297/en/. Acesso em: 07 de Janeiro de 2017. TONON, L.M. Análise Farmacoeconômica do Tratamento do Câncer Colorretal Metastático com Bevacizumabe no Brasil. Dissertação. São Paulo. 2007.

\section{AGRADECICMENTOS \\ DESiD - Ministério da Saúde.}

